\title{
Stochastic optimization of multi-reservoir systems with power plants and spillways
}

\author{
B. F. Lamond \& P. Lang \\ Department of Operations and Decision Systems, \\ Université Laval, Canada
}

\begin{abstract}
We examine a stochastic optimization model of a multiple reservoir water resource system in which the spilled outflows may have a different routing than the turbined outflows. We extend some results about the monotonicity of optimal decision rules, which were known for particular routings, and we show their validity for arbitrary routings of spilled outflows, provided they satisfy an intuitive monotonicity condition. Special cases are when the spilled outflows are expelled from the system, or when the spilled outflows are routed to the next reservoir downstream. The monotonicity of optimal policies and of the corresponding future value function can be exploited to develop efficient computational algorithms based on a dynamic programming methodology, especially when the rewards are given by a concave, piecewise linear function of electricity generation.

Keywords: multi-reservoir system, hydro-power production, turbined outflow routing, spilled outflow routing, arborescent network, incidence matrix, stochastic optimization model, stochastic dynamic programming, convex analysis, monotone optimal policy.
\end{abstract}

\section{Introduction}

The problem of optimizing long-term production for a hydroelectric system with multiple reservoirs is notoriously difficult, and most optimization models assume a planning horizon in which time is discretized into a finite number of fixed length intervals or periods. Due to the large variability of weather systems, the natural hydraulic inflows are represented by random variables, thus forming a stochastic process whose evolution laws are investigated by statistical analysis of time series data from historical records. Because typical inflow processes were found to 
be Markovian, the methodology of Markov decision processes (i.e., stochastic dynamic programming) is considered appropriate for the long-term planning of hydroelectric systems (see, e.g., Lamond and Boukhtouta [1]).

The usual source of revenue is from sales of the pooled electricity produced by the generators in the system. The electricity is sold in various markets, each with its own demand and unit price. Utility companies also have other means of producing electricity, such as thermal plants using fossil fuels. Any deficit in hydroelectric generation results in variable costs (mostly from fuel purchases) for operating the thermal plants. By comparison, variable costs of hydroelectric generation are negligible. Moreover, it is not possible to store electricity for future consumption, therefore any surplus production must be liquidated immediately on a spot market, usually at a discount price. Hence the profit function for hydroelectric production is the difference between total revenues from electricity sales and total fuel purchase costs.

A standard assumption is that revenues in one period are given by a concave function of total electricity production, while costs are given by a convex function of total thermal production. Profits are then a concave function of the hydroelectric energy produced. This reward function is usually represented by a piecewise polynomial function, possibly nondifferentiable at the breakpoints (see, e.g., Hanscom et al [2]). Another standard assumption is that the variance on future demands and prices can be neglected by comparison with the very large variance of the natural inflows. In this paper, we assume the one-period expected rewards are given by an increasing, concave function of the system's hydroelectric production.

Another important assumption we make is that the amount of electricity produced at any hydro-plant is proportional to the volume of water released through its turbines during the period, and that it is independent of the volume of water stored in the adjacent reservoir. When combined with the preceding paragraph, this assumption implies that the one-period reward is a concave function of the decision variables. The concavity property, in turn, is essential in the derivation of important structural properties of optimal solutions.

From a practitioner's viewpoint, this assumption may raise two objections: $(i)$ in many actual hydro-plants, turbine efficiencies vary significantly with the stored volumes, due to the effect of water head on the turbines, and ( $i i$ ) for a given head, the power response curves of the turbines are nonlinear functions of the water flow. Concerning the latter objection, it has been found that with an adequate commitment sequence for the different turbines of a same hydro-plant, the linear approximation is reasonably accurate under normal operating conditions (see, e.g., Hanscom et al [2]). Concerning the former, we note that for many large, multi-year reservoirs, the head varies by less than $5 \%$ around its mean value. Moreover, as was found in Lamond [3] and Lamond and Boukhtouta [4] for a single reservoir system, optimal decision rules of systems with modest head variations retain the main structural properties that were found in Gessford and Karlin [5] (and exploited for computations in Lamond and Lang [6]) when head effects are neglected.

We also make a standard assumption about the system's topology. Specifically, we assume the multiple reservoir system can be represented by an arborescent 


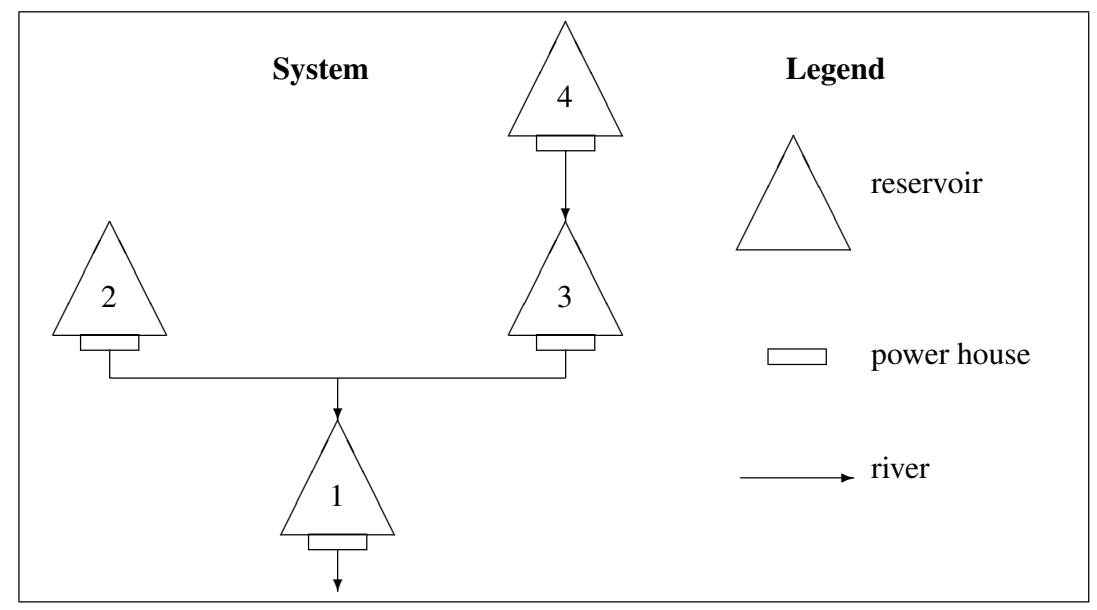

Figure 1: An arborescent network for reservoir releases.

network, as in Lamond and Sobel [7]. That is, we suppose each reservoir has exactly one outlet for its turbined flows, as in fig. 1. We also suppose there is no pumping nor interbasin transfers. Moreover, we will also show that our results remain valid for systems in which the spilled flows bypass some of the downstream reservoirs. Spillways for flood control can thus be included in the model.

In this paper, we examine the stochastic, arborescent multi-reservoir control problem with concave rewards, over a finite planning horizon, and the structure of its optimal solutions. Our main result is that under an appropriate change of variables, the optimal expected future value functions at each period are increasing and concave, provided the same is true about the terminal rewards. Archibald et al [8] also addressed the structure of optimal solutions for arborescent reservoirs, but under more restrictive hypotheses than ours. Hence their results can be seen as a particular cases of ours.

The paper is organized as follows. Basic notation and concepts from convex analysis are briefly reviewed in $\S 2$. Then in $\S 3$, we describe the arborescent multireservoir model and we explain our monotonicity assumption about the spillage routing matrix. Next, the standard equations describing reservoir dynamics are given in $\S 4$. Our stochastic optimization model is presented in $\S 5$ together with our main analytical results, followed by concluding remarks in $\S 6$.

\section{Basic notation}

Let $I$ be the identity matrix, $e_{j}$ its $j$ th column (i.e., the $j$ th unit vector) and $e$ a column vector of 1 's. Depending on context, 0 denotes the number 0 or a conformable vector or matrix of 0's. For a matrix $A$, let $A^{T}$ be its transpose, $A^{-1}$ its inverse (when it exists), $A_{\bullet j}$ its $j$ th column and $A_{i \bullet}$ its $i$ th row. For 
conformable vectors or matrices $x$ and $y$, the operators $(x)^{+}, x \wedge y$ and $x \vee y$ denote componentwise positive part, min and max, respectively. Similarly, we say that $x \leq y$ (resp. $x \geq y$ ) if $x_{i j} \leq y_{i j}$ (resp. $x_{i j} \geq y_{i j}$ ) for all $i, j$.

Now let $f(x)$ be a real function of a vector argument. Then we say that $f(x)$ is increasing if $f(x) \geq f\left(x^{\prime}\right)$ whenever $x \geq x^{\prime}$, and we say $f(x)$ is concave if

$$
f\left(\lambda x+(1-\lambda) x^{\prime}\right) \geq \lambda f(x)+(1-\lambda) f\left(x^{\prime}\right)
$$

for any scalar $\lambda$ such that $0 \leq \lambda \leq 1$. Similarly, we say that a function $g(x, y)$ of two vector arguments is jointly concave in $x$ and $y$ if

$$
g\left(\lambda x+(1-\lambda) x^{\prime}, \lambda y+(1-\lambda) y^{\prime}\right) \geq \lambda g(x, y)+(1-\lambda) g\left(x^{\prime}, y^{\prime}\right)
$$

for any scalar $\lambda$ such that $0 \leq \lambda \leq 1$. The following lemma is well known, see, e.g., Heyman and Sobel [9, pp. 525-526].

Lemma 2.1 Suppose the set $\mathcal{C}$ is convex and compact and the function $g(x, y)$ is jointly concave in $x$ and $y$ for $(x, y) \in \mathcal{C}$, and let the function $f(x)=\max _{y} g(x, y)$ subject to $(x, y) \in \mathcal{C}$. Then $f(x)$ is concave on its domain.

\section{Network structure}

Consider $n$ reservoirs, each with a hydro-plant, forming one or several arborescences. Each reservoir has one outlet into the turbine penstocks. The penstock releases either leave the system or flow into another reservoir downstream. The system can be represented by a directed graph, as in Figure 1, where each node is associated with a reservoir and each arc is associated with a release (i.e., a river). We adopt the following conventions: $(i)$ the nodes and arcs are numbered from 1 to $n,($ ii) arc $i$ leaves node $i$, and (iii) if node $i$ is downstream from node $j$ then $i<j$. Let $B$ be the node-arc incidence matrix of the network, and for each node $i$, define the set $\mathcal{P}_{i}(B)$ of its immediate predecessors, and the set $\mathcal{Q}_{i}(B)$ containing node $i$ itself and all the nodes upstream of $i$ (i.e., all predecessors, direct and indirect). Then each column of $B$ has at most two nonzero entries: $b_{j j}=1$ and, if arc $j$ flows into another node $i<j, b_{i j}=-1$. Well-known, elementary properties of arborescent networks are summarized in the following lemma, where $\left(\right.$ iii) follows by transitivity of the relation $j \in \mathcal{Q}_{i}(B)$.

Lemma 3.1 Suppose $B$ is the incidence matrix of an arborescent network satisfying the above conventions. Then

(i) its inverse matrix $B^{-1}$ exists;

(ii) the nonzero entries of $B^{-1}$ are $b_{i j}^{-1}=1$ for all nodes $j \in \mathcal{Q}_{i}(B)$; (iii) $b_{i k}^{-1} \geq b_{i j}^{-1} b_{j k}^{-1}$ for every $i, j, k \in\{1, \ldots, n\}$.

We remark that the matrices $B$ and $B^{-1}$ are upper triangular. Moreover, $x \geq 0$ implies $B^{-1} x \geq 0$, but $B^{-1} x \geq 0$ does not imply $x \geq 0$. Special cases of particular interest are the parallel system in which $B=B^{-1}=I$, with $I$ the 
identity matrix, and the serial system in which $B$ is bidiagonal with $b_{i, i+1}=-1$, and $b_{i j}^{-1}=1$ for $j=i, \ldots, n$. For the network of fig. 1 , we have

$$
B=\left(\begin{array}{cccc}
1 & -1 & -1 & 0 \\
0 & 1 & 0 & 0 \\
0 & 0 & 1 & -1 \\
0 & 0 & 0 & 1
\end{array}\right) \quad \text { and } \quad B^{-1}=\left(\begin{array}{cccc}
1 & 1 & 1 & 1 \\
0 & 1 & 0 & 0 \\
0 & 0 & 1 & 1 \\
0 & 0 & 0 & 1
\end{array}\right)
$$

Now suppose vector $x$ gives the reservoir contents, with $x_{i}$ the volume of water contained in reservoir $i$. Then $\left(B^{-1} x\right)_{i}$ is the total amount of water available in storage upstream of the $i$ th power plant. Similarly, let $c_{i}$ be the $i$ th turbine efficiency (i.e., each unit volume of water released through turbines at plant $i$ produces $c_{i}$ units of electricity) and $c$ be a row vector. Then $\gamma_{j}=\left(c B^{-1}\right)_{j}$ is the total generation potential of one unit of water stored in reservoir $j$, taking into account all downstream power plants. Then the function

$$
\gamma(x)=\gamma x=c B^{-1} x
$$

gives the total potential energy stored in the system.

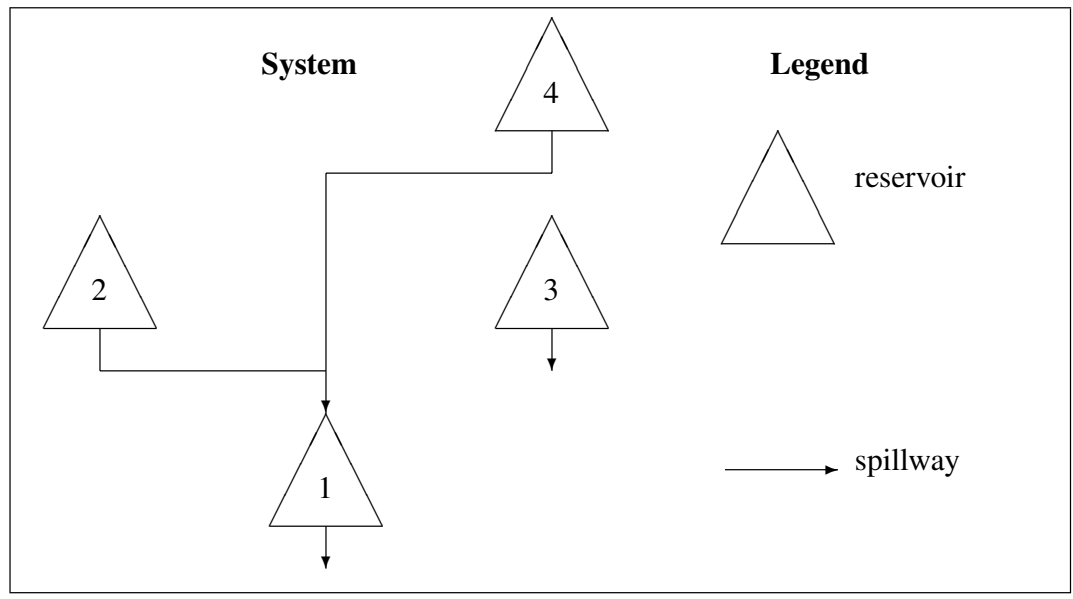

Figure 2: An arborescent network for spillways.

Now in addition to the turbine penstocks, each reservoir has a sluice that evacuates excess water without electricity generation. At many sites, the spilled water then flows on the same river as the turbined flows, to the next reservoir downstream. At some sites where floods occur frequently, however, a spillway diverts the water out of the system. For example, at the High Assouan dam in Egypt, a spillway diverts flood water into a desert valley where it evaporates (see, e.g., Georgakakos [10]). Our model allows each reservoir to have a spillway that diverts the spilled water according to one of three possibilities: $(i)$ bypass the 
power plant and flow into the next reservoir downstream, (ii) bypass the power plant and flow into another reservoir, further downstream, and (iii) bypass the power plant and flow directly out of the system. The spillways then form another arborescent network, e.g., as in fig. 2, where the spillway for reservoir 2 satisfies rule $(i)$, that of reservoir 3 satisfies ( iii) and the spillway for reservoir 4 satisfies (ii).

Let $C$ be the incidence matrix of the spillway network. The above three rules are equivalent to $C^{-1} \leq B^{-1}$. Indeed, for fig. 2 , we have

$$
C=\left(\begin{array}{cccc}
1 & -1 & 0 & -1 \\
0 & 1 & 0 & 0 \\
0 & 0 & 1 & 0 \\
0 & 0 & 0 & 1
\end{array}\right) \quad \text { and } \quad C^{-1}=\left(\begin{array}{cccc}
1 & 1 & 0 & 1 \\
0 & 1 & 0 & 0 \\
0 & 0 & 1 & 0 \\
0 & 0 & 0 & 1
\end{array}\right) \leq B^{-1}
$$

Special cases are $C=B$ when all spillways are of type $(i)$, and $C=I$ when all spillways are of type (iii). Other cases with $C^{-1} \leq B^{-1}$ can be seen as intermediate to these two extreme cases. Most models in the literature assume one of the two extremes (e.g., $C=B$ in Archibald et al [8], and $C=I$ in Lamond and Sobel [7]). The results derived in this paper are valid for the two extreme cases and for all intermediate topologies as well. The following inequalities are useful in the sequel.

Lemma 3.2 Suppose $B$ and $C$ are incidence matrices such that $C^{-1} \leq B^{-1}$ and let $x$ be an arbitrary column vector. Then

(i) $B^{-1} C \geq I$;

(ii) $C^{-1} x \geq 0 \Rightarrow B^{-1} x \geq 0$.

Proof. $(i)$ There are two cases.

Case 1: $C_{\bullet j} \geq B_{\bullet j}$. Then $B^{-1}\left(C_{\bullet j}-B_{\bullet j}\right) \geq 0$, i.e., $B^{-1} C_{\bullet j} \geq e_{j}$.

Case 2: $C_{\bullet j} \nsupseteq B_{\bullet j}$. Then $C_{\bullet j}=e_{j}-e_{i}$ for some $i<j$ such that $j \in \mathcal{Q}_{i}(B)$, and $B_{k \bullet}^{-1} C_{\bullet j}=b_{k j}^{-1}-b_{k i}^{-1}$ for every $k \in\{1, \ldots, n\}$. By Lemma 3.1( $\left.i i i\right)$, we have $b_{k j}^{-1} \geq b_{k i}^{-1} b_{i j}^{-1}$ which implies $b_{k j}^{-1} \geq b_{k i}^{-1}$ because $b_{i j}^{-1}=1$, hence $B_{k \bullet}^{-1} C_{\bullet j} \geq 0$. In the special case $k=j$, we have further that $b_{k i}^{-1}=0$ because $B^{-1}$ is upper triangular, hence $B_{k \bullet}^{-1} C_{\bullet j}=1$.

(ii) Let $z=C^{-1} x \geq 0$. From $(i), B^{-1} x=B^{-1} C z \geq z \geq 0$.

We now interpret these properties. For $i=1, \ldots, n$, let $x_{i}$ be the volume of water stored in reservoir $i$, and suppose a volume $w_{i}$ of water is released through the turbines. Then the new volumes $y_{i}$ are given by $y=x-B w$. Also, $w=B^{-1}(x-y)$ is the release vector that changes the storages from $x$ to $y$. Obviously, such a change is possible only if $w \geq 0$. Hence Lemma 3.2(ii) states that when a displacement from $x$ to $y$ is possible through the spillways (network matrix $C$ ), the same displacement is also possible through turbined releases (network matrix $B$ ). Now Lemma 3.2(i) implies $B^{-1} C w \geq w$ when $w \geq 0$. Suppose $w$ represents water evacuated through the spillways. Then $C w$ gives the net displacement in reservoir contents, and $B^{-1} C w$ gives the penstock releases required for attaining the same displacement. The lemma states that these penstock releases must be greater than or equal to the corresponding spillages. 


\section{Reservoir dynamics}

To describe the system dynamics, we suppose the planning horizon $[0, T]$ is discretized into $t$ equal intervals, and we refer to the interval $[t-1, t)$ as period $t$. Typical intervals could be quarters, months or weeks. We assume water travel times between all sites are short enough so that all discharges and spillages can be completed before the end of the period in which they are initiated. At the beginning of period $t$, the state of the system is observed. We assume the state $s_{t} \in \mathbb{R}^{n}$ is the column vector of reservoir contents in volumetric units. Conceptually, we model the system's operation in period $t$ as a sequence of three successive steps: release, inflow and spill. In practice, of course, all three events would be spread throughout the period, and their detailed planning would be performed from finer models, based on target values provided by the long-term model.

Actual hydroelectric generation takes place during the release step. The vector $z_{t}$ of turbine releases (in volumetric units) is selected, according to a decision rule, as a function of the state $s_{t}$. It is convenient to define the vector

$$
a_{t}=s_{t}-B z_{t}
$$

of stored volumes after turbine releases. Next, during the inflow step, the stored volumes are increased from the receipt of natural inflows, through the realization $d_{t}$ of the random vector $D_{t}$ of inflow volumes, where we assume independance of $D_{1}, \ldots, D_{t}$. Finally, during the spill step, all stored volumes in excess of reservoir capacities are evacuated through the spillways. Let $w_{t}$ be the vector of spilled volumes. Then

$$
s_{t+1}=a_{t}+d_{t}-C w_{t}
$$

gives the stored volumes at the beginning of period $t+1$, where a new cycle begins.

The purpose of an optimization model is to find the decision rules to use in the release and spill steps. The decision rule for period $t$ must specify the turbine release vector $z_{t}$, or equivalently, the stored volumes $a_{t}$ after turbine releases, as a function of the observed state $s_{t}$. Assuming the reservoir capacities are limited and noting that $z_{t} \geq 0$ and eqn (2) is equivalent to $z_{t}=B^{-1}\left(s_{t}-a_{t}\right)$, the action variables must satisfy the constraints

$$
B^{-1}\left(s_{t}-a_{t}\right) \geq 0 \quad \text { and } \quad 0 \leq a_{t} \leq U
$$

where $U_{i}$ is capacity of reservoir $i, i=1, \ldots, n$. Similarly for the spillages, we have $w_{t} \geq 0$ and eqn (3) gives $w_{t}=C^{-1}\left(a_{t}+d_{t}-s_{t+1}\right)$ so that the next state variable must satisfy the constraints

$$
C^{-1}\left(a_{t}+d_{t}-s_{t+1}\right) \geq 0 \quad \text { and } \quad 0 \leq s_{t+1} \leq U
$$




\section{Stochastic optimization}

Now, for the purpose of optimization, we find it convenient to replace the state and action variables $s_{t}$ and $a_{t}$, respectively, by

$$
x_{t}=C^{-1} s_{t} \quad \text { and } \quad y_{t}=C^{-1} a_{t} .
$$

Then we assume that, during each period $t, t=1, \ldots, T$, a reward is earned depending on the observed state $x_{t}$ and the action taken $y_{t}$. As mentioned in the introduction, we suppose this reward is a concave function $g_{t}\left(E_{t}\right)$ of the energy $E_{t}$ produced during the period which, according to eqn (1), is given by

$$
E_{t}=\gamma\left(s_{t}\right)-\gamma\left(a_{t}\right)=c B^{-1}\left(s_{t}-a_{t}\right)=c B^{-1} C\left(x_{t}-y_{t}\right) .
$$

We suppose also a terminal reward $V_{T+1}\left(x_{T+1}\right)$ is associated with the residual water storages at the end of the planning horizon, and we assume future rewards are discounted with a discount factor $\beta$ such that $0 \leq \beta \leq 1$. Now for $t=1, \ldots, T$, let $V_{t}\left(x_{t}\right)$ be the optimal expected cumulative discounted reward from period $t$ to the end of the planning horizon. In order to define a dynamic programming (DP) recursion for $V_{t}\left(x_{t}\right)$, we will proceed in two steps.

In the first step, we suppose the action $y_{t}$ is known and the inflows $d_{t}$ have been observed, so that the spillages have to be determined so as to maximize future rewards. To do this, we define the function

$$
\begin{aligned}
h_{t}\left(y_{t}, d_{t}\right)=\max _{x_{t+1}} & V_{t+1}\left(x_{t+1}\right) \\
\text { s.t. } & x_{t+1} \leq y_{t}+C^{-1} d_{t} \\
& 0 \leq C x_{t+1} \leq U
\end{aligned}
$$

where the constraints (9) and (10) were obtained from (5) using eqn (6).

In the second step, we take expectations with respect to the random inflows $D_{t}$, which for simplicity we assume to have a finite distribution, giving

$$
W_{t}\left(y_{t}\right)=\mathbf{E}\left[h_{t}\left(y_{t}, D_{t}\right)\right]
$$

and next we find the action $y_{t}$ to maximize the sum of rewards for electricity produced in the current period, $g_{t}\left(E_{t}\right)$ with $E_{t}$ in eqn (7), plus discounted expected future rewards of eqn (11). Doing this, we obtain

$$
\begin{gathered}
V_{t}\left(x_{t}\right)=\max _{y_{t}} g_{t}\left(c B^{-1} C\left(x_{t}-y_{t}\right)\right)+\beta W_{t}\left(y_{t}\right) \\
\text { s.t. } \quad B^{-1} C y_{t} \leq B^{-1} C x_{t} \\
0 \leq C y_{t} \leq U
\end{gathered}
$$

where the constraints (13) and (14) were obtained from (4) using eqn (6).

The DP recursion then starts with the given function $V_{T+1}\left(x_{T+1}\right)$ of terminal rewards, and proceeds by solving successively the functions $h_{t}\left(y_{t}, d_{t}\right)$, then 
$W_{t}\left(y_{t}\right)$ and $V_{t}\left(x_{t}\right)$, for $t=T, T-1, \ldots, 1$. If the reservoir system's only economic activity is hydroelectric production, then it is reasonable to assume the terminal reward is an increasing function of the terminal state $x_{T+1}$. Moreover, under our previous assumptions about linear electricity generation and concave reward function, concavity of the terminal reward function is also plausible.

Theorem 5.1 Suppose the function $V_{T+1}\left(x_{T+1}\right)$ is increasing and concave. Then the functions $V_{t}\left(x_{t}\right)$ are increasing and concave, for $t=1, \ldots, T$.

Proof. We proceed by backward induction, showing the property holds for $V_{t}\left(x_{t}\right)$ if it holds for $V_{t+1}\left(x_{t+1}\right)$. Concavity of $h_{t}\left(y_{t}, d_{t}\right)$ with respect to $y_{t}$ for fixed $d_{t}$ follows by Lemma 2.1 from concavity of the objective function of eqn (8) and convexity of the set of points $\left(x_{t}, y_{t}\right)$ satisfying inequalities (9) and (10). Increasingness follows because if $x_{t+1}$ is feasible for some $y_{t}$ then it is also feasible for any $y_{t}^{\prime} \geq y_{t}$. Next, $W_{t}\left(y_{t}\right)$ is increasing and concave because it is equal to a sum of increasing and concave functions. Then, concavity of $V_{t}\left(x_{t}\right)$ follows by Lemma 2.1 from joint concavity of the objective function of eqn (12) in $x_{t}$ and $y_{t}$ and convexity of the set of points $\left(x_{t}, y_{t}\right)$ satisfying inequalities (13) and (14). Finally, increasingness follows from the fact that if $y_{t}$ is feasible in (13) and (14) for some $x_{t}$ then it is also feasible for any $x_{t}^{\prime} \geq x_{t}$. To show this, let $x_{t}^{\prime}=x_{t}+\delta$ where $\delta \geq 0$. Then

$$
B^{-1} C x_{t}^{\prime}=B^{-1} C x_{t}+B^{-1} C \delta \geq B^{-1} C x_{t}
$$

because Lemma 3.2( $i$ ) implies $B^{-1} C \delta \geq \delta \geq 0$.

Corollary 5.2 The optimal spillages are given, for $i=n, n-1, \ldots, 1$, by

$$
w_{i t}^{*}=\left\{a_{i t}+d_{i t}-U_{i}+\sum_{j \in \mathcal{P}_{i}(C)} w_{j t}^{*}\right\}^{+}
$$

and the optimal solution of eqns (8) to (10) is $x_{t+1}^{*}=y_{t}+C^{-1} d_{t}-w_{t}^{*}$.

Proof. $\left(I, C^{T}\right)$ being a Leontieff matrix, it follows from Cottle and Veinott [11, Theorem 2] that the feasible set of constraints (9) and (10) contains a maximal element $x_{t+1}^{*}$ or, equivalently, a minimal spillage $w_{t}^{*}$ given by eqn (15). The resulting reservoir contents $s_{t+1}^{*}$ are then given by eqn (3), with corresponding state variable $x_{t+1}^{*}$ as above. This solution is maximal in the sense that $x_{t+1}^{*} \geq x$ for all feasible $x$, and increasingness of $V_{t+1}\left(x_{t+1}\right)$ implies its optimality in eqn (8).

\section{Concluding remarks}

Although the spillages are easily obtained by eqn (15), finding optimal decision rules for the electricity generation problem (12)-(14) remains a computational challenge. The particular structure presented in Archibald et al [8] for $C=B$ and piecewise linear rewards $g_{t}\left(E_{t}\right)$ is easily shown to hold whenever $C^{-1} \leq B^{-1}$, but it is destroyed when other constraints are added to (13) and (14), such as upper limits on turbine generation capacities. By contrast, Theorem 5.1 still holds when new constraints are added, and is not restricted to piecewise linear rewards. It 
could also be extended to the case when the natural inflows are autocorrelated, by augmenting the state space with a hydrologic component, such as in Turgeon [12].

\section{Acknowledgements}

This research was supported in part by the National Science and Engineering Research Council of Canada, under Grants 0105560 and 0121330, and the Fonds pour la Formation de Chercheurs et l'Aide à la Recherche du Québec, under Grant 1570 .

\section{References}

[1] Lamond, B.F. \& Boukhtouta, A., Water reservoir applications of Markov decision processes. Handbook of Markov Decision Processes: Methods and Applications, eds. E. A. Feinberg \& A. Schwartz, Kluwer, pp. 537-558, 2001.

[2] Hanscom, M. A., Lafond, L., Lasdon, L. \& Pronovost G., Modeling and resolution of the medium term generation planning problem for a large hydro-electric system. Management Science, 26, pp. 659-668, 1980.

[3] Lamond, B. F., Stochastic optimization of a hydroelectric reservoir using piecewise polynomial approximations. INFOR, 41(1), pp. 51-69, 2003.

[4] Lamond, B. F. \& Boukhtouta, A., Neural approximation for the optimal control of a hydroplant with random inflows and concave revenues. ASCE J. of Energy Engineering, 131(1), pp. 72-95, 2005.

[5] Gessford, J. \& and Karlin, S., Optimal policy for hydroelectric operations. Studies in the Mathematical Theory of Inventory and Production, Stanford University Press: Stanford, Calif., pp. 179-200, 1958.

[6] Lamond, B. F. \& Lang, P., Lower bounding aggregation and direct comput. for an infinite horizon one-reservoir model. EJOR, 95, pp. 404-410, 1996.

[7] Lamond B. F. \& Sobel, M. J., Exact and approximate solutions of affine reservoir models. Operations Research, 43(5), 771-780, 1995.

[8] Archibald, T. W., Buchanan, C. S., Thomas L. C. \& McKinnon, K. I. M., Controlling multi-reservoir systems. EJOR, 129, pp. 619-626, 2001.

[9] Heyman, D. P. \& Sobel, M. J., Stochastic Models in Operations Research, Vol. II, McGraw-Hill: New York, 1984.

[10] Georgakakos, A. P., Extended LQG control for the real time operation of reservoir systems. Dynamic Programming for Optimal Water Resources Systems Analysis, ed. A. O. Esogbue, Prentice-Hall, pp. 329-360, 1989.

[11] Cottle, R. W. \& Veinott, A. F. Jr., Polyhedral sets having a least element. Mathematical Programming, 3, pp. 238-249, 1972.

[12] Turgeon, A., Solving a stochastic reservoir management problem with multilag autocorrelated inflows. Water Resources Research, 41:12, pp. 1-9, 2005. 